\title{
Accurate quantification of selenoproteins in human plasma/serum by isotope dilution ICP-MS: Focus on Selenoprotein P
}

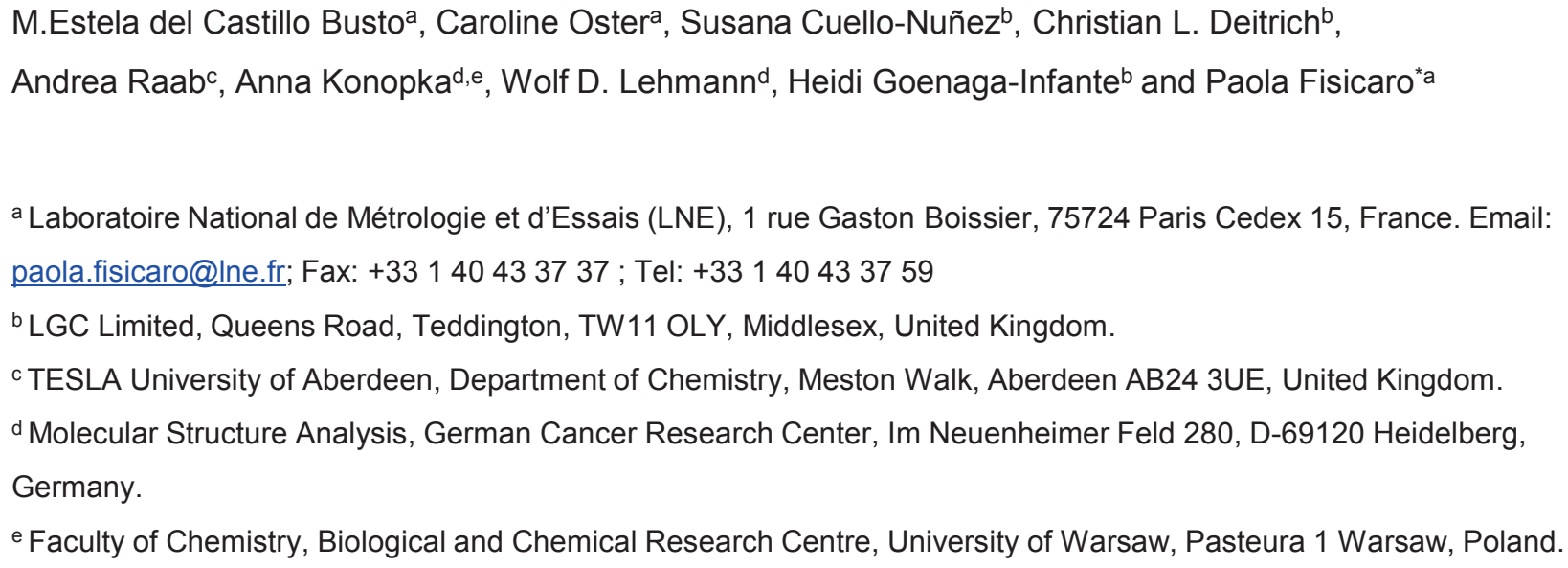

This work describes a systematic approach to the accurate quantification of selenoproteins (SEPP1) in human plasma/serum by IDA-LC-ICP-MS

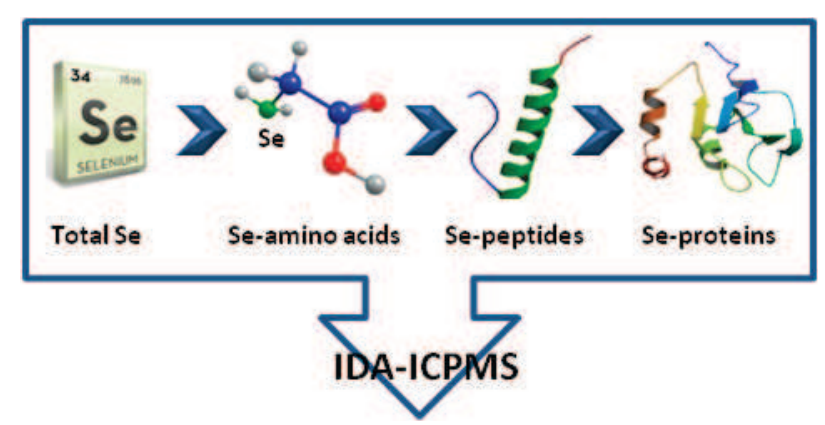




\section{Accurate quantification of selenoproteins in human plasma/serum by isotope dilution ICP-MS: Focus on Selenoprotein P}

M.Estela del Castillo Busto ${ }^{a}$, Caroline Oster ${ }^{a}$, Susana Cuello-Nuñez ${ }^{b}$, Christian L. Deitrich ${ }^{b}$, Andrea Raab ${ }^{c}$, Anna Konopka ${ }^{\text {d,e }}$, Wolf D. Lehmann ${ }^{d}$, Heidi Goenaga-Infante ${ }^{b}$ and Paola Fisicaro $^{* a}$

a Laboratoire National de Métrologie et d'Essais (LNE), 1 rue Gaston Boissier, 75724 Paris Cedex 15, France. Email: paola.fisicaro@Ine.fr; Fax: +33140433737 ; Tel: +33140433759

${ }^{\mathrm{b}}$ LGC Limited, Queens Road, Teddington, TW11 OLY, Middlesex, United Kingdom.

${ }^{\mathrm{c}}$ TESLA University of Aberdeen, Department of Chemistry, Meston Walk, Aberdeen AB24 3UE, United Kingdom.

${ }^{d}$ Molecular Structure Analysis, German Cancer Research Center (DKFZ), Im Neuenheimer Feld 280, 69120 Heidelberg, Germany.

${ }^{\mathrm{e}}$ Faculty of Chemistry, Biological and Chemical Research Centre, University of Warsaw, Pasteura 1, 02-093 Warsaw, Poland. 


\begin{abstract}
A species-specific isotope dilution analysis (SS IDA) method was developed for the first time for the determination of selenoprotein P (SEPP1) in human plasma/serum at the protein level by double affinity HPLC-ICP-MS. In this regard, a standard and a spike of SEPP1 were produced by cell-free E.coli protein synthesis, where Se (ICP tag) was introduced in the form of selenomethionine (SeMet) allowing for the absolute SEPP1 quantification by ICP-MS. A complete characterization of the standard and the spike was carried out in terms of isotopic composition and Se mass fraction by collision-cell ICP-MS to ensure SI-traceability. Method development and validation were conducted using the reference materials BCR-637, extensively analysed for its Se species, and the SRM 1950, which provides reference values for Se species (including SEPP1). Stability of the isotope ratio $\mathrm{R}_{77 / 76}$ in the sample blends was tested for one month with negligible change. Relative expanded uncertainties of $5.7 \%$ and $7.7 \%$ were achieved for BCR-637 and SRM 1950 for mass fractions of 55.5 and $63.9 \mathrm{ng}$ $\mathrm{g}^{-1}$ Se for SEPP1, respectively. The developed SEPP1 SS IDA methodology could be a valuable tool to establish references values for selenoproteins in clinical chemistry and showed the potential of cell-free protein synthesis for the preparation of future stable isotopelabeled intact selenoproteins.
\end{abstract}

Key-words: selenoproteins, SEPP1, isotope dilution, ICP-MS, BCR-637, SRM 1950. 


\section{Introduction}

Selenium (Se) is an essential nutrient (recommended daily allowance of $55 \mu \mathrm{g} / \mathrm{day}$ ) and trace element for human health ${ }^{1}$. The most common Se-species in food are the inorganic selenite and selenate as well as the Se-amino acids selenocysteine (SeCys) and selenomethionine (SeMet, the major dietary form). The health benefits of $\mathrm{Se}$ in humans come from its antioxidant properties, its involvement in the immune and endocrine system and its protection against certain disorders, in particular cancer ${ }^{2}$. Randomized controlled trials of Se supplementation for cancer prevention have yielded conflicting results, pointing out the complexity of Se metabolism due to the different toxicological and physiological properties of Se-species $1^{3}$. Thus, a better understanding of Se speciation is required. The biological activity of $\mathrm{Se}$ is principally mediated by selenoproteins, where $\mathrm{Se}$ is genetically incorporated as SeCys. The human selenoproteome contains 25 known selenoproteins, and among these selenoprotein P (SEPP1) and plasma glutathione peroxidase (GPX3) are the most abundant Se-species in human plasma/serum. SEPP1, which accounts for about $50 \%$, is responsible for Se transport and homeostasis, while GPx3 is an antioxidant enzyme which constitutes approximately $15-20 \%$ of total Se. The other main plasma/serum form of Se is Se-human serum albumin (Se-Albumin), a Se-containing protein, which occurs by the non-specific replacement of methionine with SeMet ${ }^{2}$. These three proteins (GPx3, SEPP1 and SeAlbumin) are the most commonly used markers for the assessment of Se status in human plasma/serum. However, its accurate determination and method validation remains a difficult task because of the lack of speciated certified reference materials (CRMs), pure primary standards and reference methods.

Isotope dilution mass spectrometry (IDMS) is recognised by the CCQM (Consultative Committee on Amount of Substance) as a primary measurement procedure ${ }^{4}$, i.e. a method which is completely described and understood, having the highest metrological qualities and for which the results can be given with a complete uncertainty statement. The combination of isotope dilution analysis (IDA) with ICP-MS can lead to results with the highest accuracy, precision and smallest combined uncertainty. Therefore, it is the method commonly used by most of the National Metrology Institutes (NMIs) for reference material (RM) certification or used as a reference method for elemental and speciation analysis ${ }^{5}$. IDA-ICP-MS has been used for the certification of the total Se content (64-133 $\left.\mathrm{ng} \mathrm{g}^{-1}\right)$ in the following human serum/plasma RMs: BCR 637-9, ERM-DA120a and SRM 1950. This was rather easy to achieve due to the availability of commercially isotopically-enriched Se spikes (i.e. ${ }^{74} \mathrm{Se},{ }^{76} \mathrm{Se}$, ${ }^{77} \mathrm{Se}$, etc.). However, the accurate quantification of selenoproteins using hyphenated techniques (speciation approach) has been limited by the non-availability of commercially species-specific enriched intact selenoproteins. As an alternative, Jitaru et al. ${ }^{6}$ developed a species-specific (SS) IDA-ICP-MS approach at the amino-acid level for the determination of 
Se-Albumin based on the enzymatic hydrolysis of the serum, in the presence of ${ }^{76} \mathrm{Se}$ isotopically enriched in SeMet, and the quantification of the released SeMet by ion paring reversed-phase ICP-MS. However, SeMet quantification can be only correlated to an accurate Se-Albumin determination after proving the absence of free SeMet in the serum. On the other hand, it allows the simultaneous determination of SeCys ${ }^{7}$, but it is not able to distinguish between GPx3 and SEPP1, since both contain SeCys. Alternative quantification approaches at the protein level by on-line external calibration ${ }^{6,8-10}$, antibodies ${ }^{11,12}$ and species-unspecific IDA-ICP-MS ${ }^{9,13-18}$ have been conducted in order to overcome the lack of pure Se-proteins standards and spikes. Among these quantification methods, the most accurate approach has been species-unspecific IDA-ICP-MS (also called post-column IDA), which allows the quantification of Se in Se-species when they are unknown or when Seprotein standards are not available, by the post-column addition of isotopically enriched Se spikes (i.e. ${ }^{77} \mathrm{Se},{ }^{74} \mathrm{Se}$ ). This methodology was used to provide reference values of GPx3, SEPP1 and Se-Albumin in the reference material SRM 1950 after the separation of Sespecies by double affinity HPLC ${ }^{14}$. Although quantitative Se recovery was proved, postcolumn IDA is not considered a primary method of analysis and it does not account for any losses or transformation occurring during sample preparation or species separation. Moreover, metrological traceability needs to be assured by the accurate determination of the mass injected, the mass flow and the mass fraction of the spike ${ }^{19}$.

In order to overcome these limitations, SS IDA approaches should be explored for the accurate determination of selenoproteins in human plasma/serum. In this regard, the aim of this work was the development of metrological methodologies based on the use of IDA in combination to LC-ICP-MS to ensure SI-traceability in Se speciation analysis. For this purpose, a SEPP1 SS IDA-ICP-MS approach was carried out for the first time by using a synthesized [Sec-to-Cys]SEPP1 stable isotope spike enriched in ${ }^{76}$ SeMet. Two human serum/plasma RMs were used for method development and accurate assessment: the BCR637, which is certified for total Se and widely analysed for its Se species by other authors, and the SRM 1950, which provides reference values for total Se and Se species. In order to achieve a complete mass balance of $\mathrm{Se}$ in these human plasma/serum RMs, the total $\mathrm{Se}$ content was determined by IDA-ICP-MS and Se species (GPx3, SEPP1 and Se-Albumin) were also quantified by post-column IDA.

\section{EXPERIMENTAL}

\section{Reagents and Materials}

All reagents used were of at least analytical or high-purity grade. Ultrapure water of 18.2 $\mathrm{M} \Omega . c m$ was obtained by means of a Milli-Q system (Millipore SAS, Molsheim, France). HPLC Mobile phases containing (A) $0.05 \mathrm{M}$ and (B) $1.50 \mathrm{M}$ ammonium acetate (Sigma-Aldrich, St. 
Louis, MO, USA) were adjusted to $\mathrm{pH} 7$ with acetic acid (Merck, Darmstadt, Germany) and filtered through $0.22 \mu \mathrm{m}$ (Sigma-Aldrich). Amicon Ultra $0.5 \mathrm{~mL}$ centrifugal filters of 3 and 30 $\mathrm{kDa}$ cut-off were purchased from Sigma-Aldrich. Enriched ${ }^{77} \mathrm{Se}$ (lot k2781) was obtained from Euriso-top (Saint-Aubin, France) as elemental powder (99.20\%) and it was dissolved in a minimum volume of nitric acid suprapur ${ }^{\circledR}\left(\mathrm{HNO}_{3}\right.$, Merck) and further diluted with Milli-Q. For total Se analysis and $\mathrm{Br} / \mathrm{Se}$ hydride corrections, a primary Se standard solution SRM 3149 (NIST, Gaithersburg, Maryland, USA), a high purity Se metal (Alfa Aesar, Karlsruhe, Germany) and a certified standard solution of $1000 \mathrm{mg} \mathrm{kg}^{-1}$ of sodium bromide from SigmaAldrich were used. Intermediate and working elemental solutions were prepared in $2 \%(\mathrm{v} / \mathrm{v})$ $\mathrm{HNO}_{3}$ for direct ICP-MS measurements and in mobile phases for selenoproteins determination. SeMet (Sigma-Aldrich) and the RM SeMet enriched with ${ }^{76}$ SeMet (LGC7330) from LGC Standards (Teddington, UK) were used for the preparation of SEPP1 standard and SEPP1 spike, respectively. A nuclease-free water e.g. UltraPure ${ }^{\mathrm{TM}}$ DNase/RNase-Free distilled water (Life Technologies' ${ }^{\mathrm{TM}}$, Germany), the RTS 500 Proteomaster E.coli HY Kit (5PRIME, Germany) and the modified pEXP2- DEST Gateway ${ }^{\circledR}$ Destination Vector (Thermo Scientific) as expression vector were used during the cell-free protein synthesis.

The biological reference materials analysed in this work were: the human serum CRM BCR637 (Lot 744 and 974) from the Institute for Reference Materials and Measurements (IRMM, Geel, Belgium) and the human plasma SRM 1950 Metabolites in Human Plasma from NIST.

\section{Instrumentation}

Solutions were prepared gravimetrically in a class 10,000 cleanroom using calibrated analytical balances with 0.1 and $0.001 \mathrm{mg}$ resolution (Sartorius AG, Goettingen, Germany). A densimeter (Anton Paar, Graz, Austria) was used to determine the density of the serum. Sample digestions were carried out with a microwave oven Discover SP-D (CEM Corporation, Matthews, USA). Serum was ultrafiltrated by using a centrifuge Eppendorf 5418 (Thermo Fisher Scientific, Hamburg, Germany). The cell-free synthesis was carried using a thermomixer equipped with an RTS 500 Adapter (5PRIME) and electro-elution from polyacrylamide gel was done using an electro-eluter model 422 from Bio-Rad. The molecular characterization of human recombinant SEPP1 standard and spike were conducted by ultrahigh performance liquid chromatography electrospray mass spectrometry (UPLC ESI MS/MS) using an EASY-nLC 1000 (Thermo Scientific) coupled to an Orbitrap Velos mass spectrometer (Thermo Scientific) ${ }^{22}$. Two PEEK chromatographic columns $(5 \mathrm{~cm} \times 4.6 \mathrm{~mm}$ i.d.) were packed with $1 \mathrm{~mL}$ affinity stationary phases of commercial columns (GE Healthcare, Uppsala, Sweden) named HiTrap® Heparin HP and HiTrap® Blue HP for selenoprotein separation. The columns were connected to a Spectra System P4000 HPLC system (Thermo Fisher Scientific Inc., MA, USA) and a 6-port 2-position microelectric valve (Valco 
Instruments Co. Inc., Texas, USA) for column switching. The post-column solution was introduced through a T-connector (Supelco, Bellefonte, PA, USA) using a peristaltic pump Minipuls 3 (Gilson, Villiers, France). The HPLC system was connected directly to the ICP-MS by using a PEEK tubing (0.50 mm i.d., Supelco). A Thermo Scientific ICAP Q ICP-MS (Thermo Fisher Scientific, Bremen, Germany) was used, operating in He-KED (kinetic energy discrimination) by using $1.5 \mathrm{~mL} \mathrm{~min}^{-1} \mathrm{He}$ and $2.0 \mathrm{~mL} \mathrm{~min}^{-1} \mathrm{H}_{2}$. Operational conditions and data acquisition parameters are given in Table 1.

\section{Procedures}

Total Selenium quantification by IDA-ICP-MS. For the determination of total Se by IDA, samples containing an appropriate amount of ${ }^{77}$ Se enriched spike were microwave digested in the presence of $2 \mathrm{~g} \mathrm{HNO}_{3}$ and $3 \mathrm{~g}$ Milli-Q water, heated up to $200^{\circ} \mathrm{C}$ in 4 min and kept at this temperature for $3 \mathrm{~min}$. The extracts were further diluted with $2 \% \mathrm{HNO}_{3}$ and analysed in a quadrupole ICP-MS using a mixture of $\mathrm{He}$ and $\mathrm{H}_{2}$ to eliminate argon-based interferences. Signal intensities were corrected for Se hydration $\left(\mathrm{SeH}^{+}\right)$applying the mathematical equations previously described ${ }^{20}$ and detector dead time. The measured ${ }^{78} \mathrm{Se} /{ }^{77} \mathrm{Se}$ ratio $\left(\mathrm{R}_{78 / 77}\right)$ was then corrected for mass bias using the linear model. Double IDA-ICP-MS was applied for the determination of Se in 4 independent sample blends from BCR-637 and SRM 1950. The isotopic composition of the ${ }^{77}$ Se-enriched spike was determined by ICP-MS over 1 year and its mass fraction was calculated daily by reverse-IDA against a pure Se standard using 4 calibration blends. Plasma conditions and acquisition parameters are reported in Table 1.

Separation of selenoproteins by double affinity HPLC. The simultaneous fractionation of the selected selenoproteins (GPX3, SEPP1 and Se-Albumin) in human plasma/serum was carried out by double affinity chromatography (AF) HPLC using two custom-made columns of Heparin and Blue affinity resins, following the experimental setup described elsewhere 9 and the chromatographic conditions of Table 1.

Quantification of selenoproteins by double AF-HPLC-ICP-MS. Two IDA-LC-ICP-MS approaches, specially focused on SEPP1's quantification, were investigated for the determination of selenoproteins in the human serum/plasma RMs BCR-637 and SRM 1950. Plasma conditions and acquisition parameters for AF-HPLC-ICP-MS are reported in Table 1. The chromatographic peaks were integrated using Origin 7.5 (v7.5853, Origin Lab Corporation, Northampton, MA, USA).

Species-specific Isotope Dilution Analysis (SS IDA) for SEPP1. In order to perform SS IDA, a full-length human recombinant selenoprotein P (SEPP1_HUMAN; UniProt accession no. P49908) spike and standard were produced using the RTS 500 Proteomaster E.coli HY Kit 
according to the protocol provided by manufacturer. As an expression vector the modified pEXP2-DEST Gateway ${ }^{\circledR}$ Destination Vector containing coding sequence for SEEP1_HUMAN was applied. In the modified PEXP2-DEST vector all ten TGA codons present in the coding sequence for SEPP1_HUMAN, which in vivo are Sec codons, were mutated to TGT codons encoding canonical Cys using conventional molecular cloning methods. During cell-free protein E.coli synthesis, Se was introduced into the polypeptide chain in the form of SeMet or ${ }^{76}$ Se-Met for the preparation of the SEPP1 standard and spike, respectively. The cell-free synthesis was carried out for $24 \mathrm{~h}$ at $30{ }^{\circ} \mathrm{C}$ with shaking at 1000 rpm. The standard and spike were further purified by polyacrylamide gel electrophoresis separation (SDS PAGE) in combination with electro-elution. A more detailed description about the synthesis and the purification can be found elsewhere ${ }^{21,22}$.

The SEPP1 standard and spike were characterised in terms of their isotopic compositions by double AF-HPLC-ICP-MS using the acquisition parameters for isotopic composition (Table1) with an integration time of $0.04 \mathrm{~s}$ per isotope. Furthermore, the quantification of the total Se content in the [Sec-to-Cys]SEPP1 spike was performed by reverse IDA using two approaches. First, 3 replicates of about $50 \mu \mathrm{L}$ of the spike were ultrafiltrated by a $30 \mathrm{kDa}$ cutoff to eliminate possible non-retained species generated during the spike synthesis and the corresponding fractions were digested in the presence of $\mathrm{HNO}_{3}$. An appropriate amount of natural Se standard was added before the mineralisation and the Se quantification was performed by total IDA-ICP-MS. On the other hand, 5 aliquots of the untreated spike were injected in the chromatographic system, and the Se eluted in the SEPP1 fraction (5-10 min) was quantified using post-column IDA (see next section). For the determination of the Se content in the SEPP1 of the RMs by SS IDA, at least 3 sample blends (mixture of serum and spike) were prepared gravimetrically. After equilibration of the mixture during 2 hours at room temperature, a dilution step in buffer A was applied before the analysis by double AF-HPLCICP-MS. Each measurement batch included a procedural blank, a mass bias solution and three injections of the sample blend. The synthesized [Sec-to-Cys]SEPP1 standard containing natural Se abundances was used for $\mathrm{SeH}^{+}$and mass bias correction at a mass fraction similar to the SEPP1 in the sample $\left(\sim 50 \mathrm{ng} \mathrm{g}^{-1} \mathrm{Se}\right)$. The isotope ratio $\mathrm{R}_{77 / 76}$ was calculated and corrected as previously described.

Post-column Isotope Dilution Analysis. Secondly, a species-unspecific IDA methodology was performed for the quantification of intact selenoproteins using the principle previously described ${ }^{23} . \mathrm{A}^{77}$ Se-enriched spike $\left(0.4-0.5 \mathrm{ng} \mathrm{g}^{-1} \mathrm{Se}\right.$ in Milli-Q) is added at a continuous flow $\left(0.1 \mathrm{~g} \mathrm{~min}^{-1}\right)$ at the end of the column, obtaining a stable ${ }^{77} \mathrm{Se}$ signal. To correct for $\mathrm{SeH}^{+}$ and $\mathrm{BrH}^{+}$interferences, standard solutions of $\mathrm{Se}$ and $\mathrm{Br}\left(100 \mathrm{ng} \mathrm{g}^{-1}\right.$ and $1 \mathrm{mg} \mathrm{g}^{-1}$, respectively) prepared in phase $A$ and $B$ were introduced post-column instead of the 
enriched spike, before and after a day analysis. Then, the isotope ratio $R_{76 / 77}$ was calculated and mass bias corrected.

Uncertainty estimation. A complete uncertainty budget was made following $\mathrm{GUM}^{24}$. The combined standard uncertainty $u_{c}$ was calculated following the propagation law of uncertainties, without variable correlations. All uncertainty values are expanded uncertainties with a coverage factor of $k=2$. A dedicated software was used for the estimation of uncertainties (Wincert version 3.13.0311.0026, Implex, France, http://www.implex.fr).

\section{Results and discussion}

\section{Determination of Se-bound SEEP1 in human plasma/serum by SS IDA down to the protein level}

The accurate determination of intact selenoproteins in human plasma/serum has been limited by the absence of speciated Se CRMs, pure standards and reference methods. In this regard, method validation has been often relied on the total Se determination of the serum assuming that all $\mathrm{Se}$ is bound to the proteins which are eluted from the chromatographic system (sum of all Se-species)8,14,15. Figure 1 summarizes the different quantitative approaches developed in this work to achieve an accurate determination of $\mathrm{Se}$ in human plasma/serum. Two RMs were used in this work: the BCR-637 and the SRM 1950. The BCR637, certified for total Se, has been widely used in the literature to assess selenoproteins concentration in human serum6 $8^{-9} 9^{10,15,16}$ and the SRM 1950 provides reference values for total Se, GPx3, SEPP1 and Se-Albumin ${ }^{14}$.

Total Se measurements (Figure 1.1) could give us preliminary information regarding the normal or deficient level of Se in humans1. However, to assess a complete Se status, speciation approaches (Figure 1.2) that allow an accurate determination of all Se-species in the serum should be used. At protein level (Figure 1.3), on-line external calibration6.8 $89^{10}$ and post-column IDA8, $9^{13-18}$ have been the mainly used quantification methodologies due to the lack of isotopically enriched Se-species required to apply SS IDA. Moreover, the latter methodologies do not fulfil the requirements of primary methods and therefore are not completely suitable for the certification of RMs. In order to overcome these limitations, a SS IDA methodology was developed and validated for the first time for the accurate quantification of the Se-bound SEPP1 at the protein level in human plasma/serum (Figure 1.3a). In this regard, a full-length human recombinant and stable isotope-labeled intact selenoprotein was synthesized by cell-free E.coli protein synthesis ${ }^{21,22}$. Since this procedure currently does not allow expressing native selenoproteins, SeCys-free selenoprotein standards were synthesized by the replacement of the originally 10 SeCys-residues (Sec) by 10 cysteines (Cys) by point mutations of nucleotide triplets coding Sec to triplets coding Cys 
in the coding sequence for selenoprotein present in the expression vector used as a template for E.coli protein synthesis. The proteins obtained in this way are named [Sec-toCys]selenoprotein standards. Since these standards do not contain Sec, selenium can be introduced in the cell-free E.coli protein synthesis medium in the form of SeMet or stable isotope enriched SeMet $\left({ }^{76}\right.$ SeMet) to generate SeMet-SEPP1 or ${ }^{76}$ SeMet-SEPP1 standards, respectively. During the protein standard synthesis, 4 SeMet residues are incorporated into [Sec-to-Cys]selenoprotein P standard stoichiometrically at the sites of canonical Met in the protein structure. The verification of successful exchange of all SeCys residues for Cys residues and the incorporation of SeMet in the amino acid sequence of [Sec-toCys]selenoprotein $P$ standard were carried out by ESI MS/MS after trypsin digestion ${ }^{22}$. Figure 2 shows the MS/MS spectrum for one of the [Sec-to-Cys]SEPP1 peptides containing the exchanged Cys residue $(\mathrm{C})$, as well as their corresponding experimental and theoretical isotopic patterns.

The current presence of a Se tag in the form of SeMet $/{ }^{76}$ SeMet allowed an absolute quantification of SEPP1 via Se-specific ICP-MS detection at the protein level. In this regard, the second step in the development of the SS IDA approach was the inorganic characterisation of synthesized [Sec-to-Cys]SEPP1 standards. Firstly, the natural and the stable isotope enriched standards were characterised in terms of isotopic composition by double AF-HPLC-ICP-MS. The isotopic enrichment of ${ }^{76} \mathrm{Se}$ in the spike was $(99.85 \pm 0.05) \%$, $(k=2)$, which agrees with the isotopic abundance of the ${ }^{76}$ SeMet spike used during the synthesis (Table 2). On the other hand, the natural isotopic abundances of Se in the nonlabeled [Sec-to-Cys]SEPP1 standard were confirmed (Table 2) and this standard was further used for mass bias correction. Secondly, the Se mass fraction in the spike was calculated by reverse IDA using two approaches: a) by an on-line quantification of the SEPP1 eluted from the double AF-HPLC system using post-column IDA and a ${ }^{77}$ Se spike, which gave a mass fraction of $(409 \pm 43) \mathrm{ng} \mathrm{g}^{-1} \mathrm{Se},(\mathrm{n}=5, k=2)$ and b) by an off-line approach where the spike was purified by a $30 \mathrm{kDa}$ cut-off membrane and the corresponding fractions were mineralized and analyzed by IDA-ICP-MS. The Se mass fraction of the higher fraction was (409.0 \pm 7.0$)$ $\mathrm{ng} \mathrm{g^{-1 }}$ Se, $(n=3, k=2)$, in good agreement with the post-column value and with lower uncertainties. On the other hand, no significant Se levels were found in the filtrate ((10.9 \pm 7.0) $\mathrm{ng} \mathrm{g}^{-1} \mathrm{Se}$ ). For further confirmation, this lower fraction was injected into the HPLC system and no Se-bound to SEPP1 was detected.

Once the stable isotope enriched spike was characterized, this material was applied for the quantification of SEPP1 in the RMs by single SS IDA-ICP-MS (Supplementary information, Equation S-1) at different days using different pre-packed AF columns. Five independent sample blends of BCR-637 (Lot 744 and Lot 974) were prepared the same day and analyzed on four different days. Each sample blend was run in triplicate and the results from all 
measurements $(n=15)$ were combined yielding a Se mass fraction of $(55.5 \pm 3.2) \mathrm{ng} \mathrm{g}^{-1} \mathrm{Se}$ $(k=2)$ for SEPP1, with a relative expanded uncertainty of $5.7 \%$. This value is in good agreement with the values previously reported in the literature $8^{, 9,15,16}$ and also with those obtained by post-column IDA in this work (Table 3). The synthesized [Sec-to-Cys]SEPP1 standard was injected into the chromatographic system between each triplicate sample blend for the correction of $\mathrm{SeH}^{+}$, giving an average yield of $(2.85 \pm 0.36) \%$ evaluated at 4 different days during $8 \mathrm{~h}$ of measurement. Also, the stability of the isotope ratio $R_{77 / 76}(0.276 \pm 0.009)$ in the sample blends was tested for one month with negligible change. Figure 2 shows a chromatogram obtained for a sample blend of BCR-637 at masses 76 and 77, demonstrating the same behaviour of the naturally present SEEP1 in the serum and the stable isotope enriched SEPP1 spike.

A complete uncertainty budget was elaborated according to the ISO/GUM guide ${ }^{24}$. The parameters taken into account for the calculation of the expanded uncertainty derived from the SS IDA-ICP-MS equation (Supplementary information, Equation S-1), including the measurement precision of the method. This last analytical parameter took into account the inter- and intra-day variability, the serum interbatch variability and the use of different homepacked affinity columns. The intra-day variability was calculated as the relative standard deviation (RSD) of $\mathrm{R}_{77 / 76}$ of a duplicate sample blend, either the same blend or different blends, on a single day. Meanwhile the inter-day variability was evaluated based on the results of the sample blends on 4 different days over a period of one month. The intra-day variability, ranged from $0.2 \%$ to $2.8 \%$, pointed out that variations within a single batch were similar than ones between different batches. On the other hand, a similar inter-day variability was obtained $(0.3 \%-3.2 \%)$, which showed no significant effect on the sample interbatch variability, the sample preparation and the use of different affinity columns. Considering the laboratory repeatability or intra-day variability, the most significant variables in the uncertainty budget for the determination of SEPP1 using single SSIDA are a) the measured isotope ratio of the sample blend $(60 \%), b)$ the Se mass fraction of the spike $(17 \%), c)$ the mass of the spike $(14 \%)$ and $d)$ the mass bias correction factor, $k(8 \%)$. As can be seen, the predominant sources of uncertainty are linked to the measurement of transient signals accounting for around $68 \%$ of the overall uncertainty, i.e. $R_{77 / 76}$ in the sample blend and $R_{77 / 76}$ in the calibration standard for mass bias correction $(k)$. Taking into account the measurement precision, which combines inter- and intra-day variabilities, a relative expanded uncertainty of $5.7 \%$ was obtained.

A similar analytical approach was conducted for SRM 1950. In this particular case, three independent sample blends were prepared and run in four different days $(n=12)$. The measured isotope ratio $R_{77 / 76}(0.351 \pm 0.013)$ in the sample blends kept also stable during the time analysis. The obtained intra- and inter-day variabilities, ranged from $0.2 \%$ to $4.5 \%$, were 
slightly higher in comparison to BCR 637 . This could be ascribed to the nature of the sample, since plasma represents a more complex specimen than serum. The mass fraction of SEEP1 (as Se) in the SRM 1950 was found to be $(63.9 \pm 4.9) \mathrm{ng} \mathrm{g}^{-1} \mathrm{Se}(k=2)$ with a relative expanded uncertainty of $7.7 \%$. A breakdown of the uncertainty budget showed the same contributing factors described above. However, in this case, due to a higher sample complexity, the measured isotope ratios in the sample blend and in the calibration standard accounted for about $75 \%$ and $10 \%$ of the total uncertainty budget, respectively.

A slightly higher value for Se (as SEPP1) was obtained by SS IDA-ICP-MS in comparison to the reference value assigned by post-column IDA ((50.2 \pm 4.3$\left.) \mathrm{ng} \mathrm{g}^{-1} \mathrm{Se}, k=2.6\right)^{14}$. Although the species-unspecific approach is known to provide accurate quantification results ${ }^{23}$, it is unable to correct for possible Se losses during the analytical procedure. Furthermore, SS IDA-ICP-MS provided low uncertainties (5.7\% and 7.7\% for BCR-637 and SRM 1950, respectively), which could be used to establish reference values to clinical trials and in the characterization/validation of the calibration standard used in the ELISA kits for SEPP1. This enhancement on the SI-traceability of the immunoassays standards will allow data comparability in the determination of SEPP1 in serum/plasma.

These data were further confirmed and validated with values obtained under the frame of the EURAMET EMRP «Metrology for metalloproteins » project where a new double SS IDAICP-MS was developed based on the use of unique stable isotope enriched Se-peptides of SEPP1 (Figure 1.4a) ${ }^{25}$. Se mass fractions for SEPP1 of $(56.2 \pm 1.7) \mathrm{ng} \mathrm{g}^{-1}$ Se and $(60.6 \pm$ 3.2) $\mathrm{ng} \mathrm{g}^{-1} \mathrm{Se}(k=2)$ in BCR-637 and SRM 1950, respectively, were obtained at the peptide level, which agree well with those obtained for the whole protein using single SS IDA-ICPMS.

\section{Selenium mass balance as quality control}

In order to evaluate Se mass balance from the double AF-HPLC-ICP-MS system to provide literature data comparison in the RMs BCR 637 and SRM 1950, total Se content by IDA-ICPMS (Figure 1.1) and Se-species quantification by post-column IDA were also explored (Figure 1.3c). The simultaneous determination of Se-species in the RMs was carried out by double AF-HPLC coupled to ICP-MS using Heparin and Blue stationary phases obtaining three chromatographic peaks. The first fraction labelled as "GP3x + non-retained species" represents all Se-species, mainly GP3x8, with no affinity to these resins, while Se-bound to SEPP1 and Se-Albumin are given in the second and third fraction, respectively. Se mass balance in the double AF-HPLC-ICP-MS will be estimated by the sum of Se mass fractions from the three chromatographic fractions divided by the total Se obtained by IDA-ICP-MS.

a) Total Se content in human plasma/serum by double IDA-ICP-MS. The second step of our validation approach was the determination of total Se by double IDA-ICP-MS 
(Figure 1.1). The BCR-637 value obtained for the measured $R_{78 / 77}(79.6 \pm 1.6) \mathrm{ng} \mathrm{g}^{-1} \mathrm{Se}$ $(k=2, n=4)$ fits perfectly with the reference value $(79 \pm 7) \mathrm{ng} \mathrm{g}^{-1} \mathrm{Se}(k=2)$, which was expressed as mass fraction taking into account its measured density $(1.0237 \pm 0.0044) \mathrm{g} \mathrm{mL}^{-}$ ${ }^{1}(k=2)$. A normalised error $\left(E_{N}\right)$ of 0.14 lower than 2 proves that there are no significant differences between the determined and the certified value. For the SRM 1950, a Se mass fraction of $(96.9 \pm 4.1) \mathrm{ng} \mathrm{g}^{-1} \mathrm{Se}(k=2, \mathrm{n}=4)$ was determined by double IDA-ICP-MS, which is also in agreement with the reference value $(105.5 \pm 3.8) \mathrm{ng} \mathrm{g}^{-1} \mathrm{Se}\left(k=2.2, \mathrm{R}_{80 / 77}\right)$ with an $\mathrm{E}_{\mathrm{N}}$ of 1.50. The $\mathrm{R}_{78 / 77}$ ratio was preferred to $\mathrm{R}_{80 / 77}$ to avoid further corrections due to the $\mathrm{BrH}^{+}$ interference on the ${ }^{80}$ Se signal ${ }^{20}$.

b) Se-species determination in human plasma/serum by post-column IDA-ICP-MS. The post-column IDA methodology has been widely used to assign Se-species values in BCR human RMs8 $9^{15,16}$, in quality control materials ${ }^{16}$, in human populations ${ }^{13,15,17,18}$ and in SRM $1950^{14}$. Until now, due to the lack of speciated selenoprotein standards, this has been the most accurate quantification method for selenoproteins. However, several variables involved in the on-line equation (Supplementary information, Equation S-2) should be carefully determined to ensure accurate and SI traceable results. In this regard, the flow of the spike was gravimetrically calibrated at the beginning and at the end of each day ( $0.11005 \pm$ $\left.0.00091) \mathrm{g} \mathrm{min}^{-1}\right)$, its Se mass fraction was determined daily by reverse IDA-ICP-MS ((0.504 $\pm 0.017) \mathrm{ng} \mathrm{g}^{-1} \mathrm{Se}$ ) and the loop was gravimetrically calibrated to obtain the mass injected $((0.0495 \pm 0.0096) \mathrm{g})$. Seven replicates from 2 different lots of BCR-637 (Lot 744 and 974) were systematically analysed at 2 different days using 2 different home-packed AF columns in order to account for interbatch Se variability and column loading. Similarly, for SRM 1950, 11 replicates were analysed at 3 different days using 2 different pre-packed AF columns. A summary of the quantification results for BCR-637 and SRM 1950 using the post-column IDA methodology is presented in Table 3, as well as values for BCR-637 reported in the literature based on the same approach9, ${ }^{15,16}$. Regarding BCR-637, the developed post-column approach gave results for all three Se-species in close agreement with those already reported and a total Se recovery from the AF-HPLC system was achieved $((100 \pm 7) \%)$. Expanded uncertainties between $12 \%$ and $16 \%$ were obtained for all selenoproteins $(k=2)$, where the main contribution to the uncertainty budget was the measured isotope $R_{76 / 77}$ in the mixture $(60-89 \%)$. Other parameters that should be considered are the Se mass fraction of the spike (4-17\%) and the mass injected (6-22\%). Also in this case, no significant differences within experimental uncertainties were found by using different AF columns.

In case of SRM 1950, a good agreement between experimental and reference values ${ }^{14}$ was obtained by post-column IDA considering expanded uncertainties for the individual and the sum of Se species, except for the first fraction (GPx3 + non-retained species). A lower Se mass fraction $\left(R_{76 / 77}\right)$ was obtained in comparison to the reference value using the ratio 
$\mathrm{R}_{80 / 77}$. This higher value could be related to the interference of ${ }^{79} \mathrm{BrH}^{+}$on the ${ }^{80} \mathrm{Se}$ isotope. It was proved that the presence of $1 \mu \mathrm{g} \mathrm{g}{ }^{-1} \mathrm{Br}$ caused a bias of about $56 \%$ in the ${ }^{77} \mathrm{Se} /{ }^{80} \mathrm{Se}$ ratio in a solution of $20 \mathrm{ng} \mathrm{g}^{-1}$ of $\mathrm{Se}^{20}$. Nevertheless, a Se mass balance of (102 \pm 10$) \%$ ) was achieved for SRM 1950, which showed a complete Se chromatographic recovery. Expanded uncertainties between $11 \%$ and $16 \%$ were obtained for selenoproteins, similar to those obtained for BCR-637. Also here, the main contributions to the uncertainty budget were the measured isotope $R_{76 / 77}$ in the mixture (79-89\%), the Se mass fraction of the spike (4-9\%) and the mass injected (6-11\%). The main advantage of the post-column approach is that it allows the quantification of all possible Se species even if they are unknown and that it does not require any sample pre-treatment. On the other hand, losses before the complete mixing of sample and spike are not corrected for and post-column IDA provides higher uncertainties in comparison to SS IDA-ICP-MS (8.2\% vs $5.7 \%$ for BCR-637 and $12.1 \%$ vs $7.7 \%$ for SRM 1950 in the case of SEPP1). Furthermore, a careful control of all experimental parameters associated with the post-column equation should be conducted; while a small impact $(<1 \%)$ in the total uncertainty budget was observed for the spike's flow, the mass injected contributed between $4 \%$ to $22 \%$.

\section{Conclusions}

The present work illustrates the development of potential reference measurement procedures of high metrological order based on the combination of IDA and ICP-MS for the accurate assessment of Se in human serum. Here, a SEPP1 SS IDA at the protein level was developed for the first time by the use of a stable isotope enriched standard ([SectoCys]SEPP1). After the complete characterisation of the spike (for isotopic composition and Se mass fraction), SEPP1 was determined in the RMs BCR-637 and SRM 1950. Results obtained were in good agreement with those achieved by SS IDA via Se-peptides ${ }^{25}$ and by post-column IDA. However, SS IDA-ICP-MS provided the smallest uncertainties and seems less affected by Se interbatch variability, affinity column capacity and possible analyte losses. On the other hand, post-column IDA is a quantitative approach easier to be implemented and less time consuming, which allowed the simultaneously determination of Se-species in human serum/plasma RMs.

The developed SEPP1 SS IDA methodology could be used for the validation and quality assurance of routine assays and for the certification of RMs, which will ensure SI traceability in Se speciation. Moreover, this work reveals the excellent capabilities of cell-free E.coli protein synthesis for the production of other stable isotope labeled standards that can be extended to other selenoproteins (e.g. GPx3, thioredoxin reductase) $)^{22}$. 


\section{Acknowledgments}

The research leading to these results was funded by the EMRP Joint Research Project "Metrology for metalloproteins" (HLT-05 2012). The EMRP is jointly funded by the EMRP participating countries within EURAMET and the European Union.

\section{References}

${ }^{1}$ C. R. B. Rocourt and W.-H. Cheng, Nutrients, 2013, 5, 1349-1365.

${ }^{2}$ M. Roman, P. Jitaru and C. Barbante, Metallomics, 2014, 6, 25-54.

${ }^{3}$ M. Vinceti, P. Grill, C. Malagoli, T. Filippini, S. Storani, M. Malavolti and B. Michalke, J. Trace Elem. Med. Biol., 2015, 31, 1-10.

${ }^{4}$ ISO/IEC GUIDE 99:2007: International vocabulary of metrology -Basic and general concepts and associated terms (VIM), ed.: International Organisation for Standardisation, Geneva, 2007.

5 "Isotope dilution mass spectrometry", J.I. Alonso Garcia and P. Rodriguez-Gonzalez, Royal Society of Chemistry, Cambridge, UK, 2013.

${ }^{6}$ P. Jitaru, H. Goenaga-Infante, S. Vaslin-Reimann and P. Fisicaro, Anal. Chim. Acta, 2010, 657, 100-107.

7 J.R. Encinar, D. Schaumlöffel, Y. Ogra and R. Lobinski, Anal. Chem., 2004, 76, 6635-6642.

${ }^{8}$ P. Jitaru, M. Roman, C. Barbante, S. Vaslin-Reimann and P. Fisicaro, Accred. Qual. Assur., 2010, 15, 343-350.

${ }^{9}$ P. Jitaru, G. Cozzi, A. Gambaro, P. Cescon and C. Barbante, Anal. Bioanal. Chem., 2008, 391, 661-669.

${ }^{10}$ P. Jitaru, M. Prete, G. Cozzi, C. Turetta, W. Cairns, R. Seraglia, P. Traldi, P. Cesconac and C. Barbante, J. Anal. At. Spectrom., 2008, 23, 402-406.

${ }^{11}$ B. Hollenbach, N.G. Morgenthaler, J. Struck, C. Alonso, A. Bergmann, J. Köhrle and L. Schomburg, J. Trace Elem. Med. Biol., 2008, 22, 24-32.

${ }^{12}$ A. Andoh, M. Hirashima, H. Maeda and K. Hata, Nutrition, 2005, 21, 574-579.

${ }^{13}$ L. Hinojosa Reyes, J.M. Marchante-Gayón, J.I García-Alonso and A. Sanz-Medel, J. Anal. At. Spectrom., 2003, 18, 1210-1216.

${ }^{14}$ G. Ballihaut, L.E. Kilpatrick and W.C. Davis, Anal. Chem., 2011, 83, 8667-8674.

${ }^{15}$ S. Letsiou, Y. Lu, T. Nomikos, S. Antonopoulou, D. Panagiotakos, C. Pitsavos, C. Stefanadis and S.A. Pergantis, Proteomics, 2010, 10, 3447-3457.

${ }^{16}$ M. A. García-Sevillano, T. García-Barrera and J.L. Gómez-Ariza, J. Chromatogr. A, 2013, 1318, 171-9.

${ }^{17}$ M. Xu, L. Yang and Q. Wang, J. Anal. At. Spectrom., 2008, 123, 1545-1549. 
${ }^{18}$ Y-F Li, L. Hu, B. Li, X. Huang, E.H. larsen, Y. Gao, Z. Chai and C. Chen, J. Anal. At. Spectrom., 2011, 26, 224-229.

${ }^{19}$ G. Koellensperger, S. Hann, J. Nurmi, T. Prohaska and G. Stingeder, J. Anal. At. Spectrom., 2003, 18, 1047-1055.

${ }^{20}$ L. Hinojosa Reyes, J.M. Marchante-Gayón, J.I García-Alonso and A. Sanz-Medel, J. Anal. At. Spectrom., 2003, 18, 11-16.

${ }^{21}$ A. Konopka, N. Zinn, C. Wild and W.D. Lehmann, Methods Mol Biol., 2014, 1156, 337-363.

${ }^{22}$ A. Konopka, D. Winter, W. Konopka, M.E. del Castillo Busto, S. Nunez, H. GoenagaInfante, P. Fisicaro and W.D. Lehmann, J. Anal. At. Spectrom., 2016, submitted to the Speciation Analysis Themed Issue.

${ }^{23}$ M.E. del Castillo Busto, M. Montes-Bayón and A. Sanz-Medel, Anal. Chem., 2006, 78, 8218-8226.

${ }^{24}$ ISO/IEC Guide 98:1995: Guide to the expression of Uncertainty in Measurement, ed: International Organisation for Standardisation, Geneva, 1995.

${ }^{25}$ C.L. Deitrich, S. Cuello-Nuñez, D. Kmiotek, F.A. Thorma, M.E del Castillo Busto, P. Fisicaro and H. Goenaga-Infante, Anal. Chem., 2016, 88, 6357-6365. 


\section{$\underline{\text { Tables }}$}

Table 1. Operating conditions for the separation and quantification of selenoproteins in human plasma/serum

\author{
Affinity columns \\ Binding Buffer $(A)$ \\ Elution Buffer (B) \\ Injection volume \\ Flow rate \\ Gradient
}

Double AF-HPLC conditions

\section{iCAP Q ICP-Q-MS parameters}

RF Power

Carrier gas flow

Auxiliary gas flow

He gas flow

$\mathrm{H}_{2}$ gas flow

Collision cell Bias

Pole Bias

Total Se analysis ${ }^{c}$

Monitored masses for IDA

Monitored masses for isotopic composition

Replicates

Time resolved analysis (TRA) -

Species-specific IDA

Post-column IDA
HiTrap Heparin Sepharose ( $5 \mathrm{~cm} \times 4.6 \mathrm{~mm}$ id)

HiTrap Blue Sepharose $(5 \mathrm{~cm} \times 4.6 \mathrm{~mm}$ id)

$0.05 \mathrm{~mol} \mathrm{~L}^{-1}$ Ammonium Acetate, $\mathrm{pH} 7$

$1.50 \mathrm{~mol} \mathrm{~L}^{-1}$ Ammonium Acetate, $\mathrm{pH} 7$

$50 \mu \mathrm{L}(0.0495 \pm 0.0096) \mathrm{g}, k=2$

$1 \mathrm{~mL} \mathrm{\textrm {min } ^ { - 1 }}$

0-5 min, Buffer A, Position P1 (load)

5-10 min, Buffer B, Position P2 (inject)

10-15 min, Buffer B, Position P1

$1550 \mathrm{~W}$

$1.05 \mathrm{~L} \mathrm{~min}^{-1}$

$0.80 \mathrm{~L} \mathrm{~min}^{-1}$

$1.50 \mathrm{~mL} \mathrm{~min}^{-1}$

$2.00 \mathrm{~mL} \mathrm{~min}^{-1}$

$-21 \mathrm{~V}$

$-18 \mathrm{~V}$

$76,77,78(1 \mathrm{~s})^{\mathrm{a}} ; 82,83(0.4 \mathrm{~s})^{\mathrm{b}}$

$74,76,77,78,80,82,83(0.4 \mathrm{~s})$

5

$76,77,78(0.1 \mathrm{~s})^{\mathrm{a}} ; 82,83(0.04 \mathrm{~s})^{\mathrm{b}}$

$76,77,78(0.1 \mathrm{~s})^{\mathrm{a}}$

$76,77,78,79,80,81,82,83(0.04 \mathrm{~s})^{\mathrm{b}}$

${ }^{\text {a }}$ Masses measured for IDA

${ }^{b}$ Masses measured for corrections

${ }^{\mathrm{c}}$ Integration time is given in brackets 
Table 2. Isotopic composition of the synthesized [Sec-to-Cys]SEPP1 spike and standard by double AF-HPLC-ICP-MS

\begin{tabular}{ccc}
\hline & \multicolumn{2}{c}{ Abundance, $\%(\mathrm{n}=4, k=2)$} \\
\hline Se Isotopes & Spike & Standard \\
${ }^{74} \mathrm{Se}$ & $0.035 \pm 0.005$ & $0.69 \pm 0.02$ \\
${ }^{76} \mathrm{Se}$ & $99.85 \pm 0.05^{\mathrm{a}}$ & $7.7 \pm 0.3$ \\
${ }^{77} \mathrm{Se}$ & $0.05 \pm 0.06$ & $6.5 \pm 0.3$ \\
${ }^{78} \mathrm{Se}$ & $0.03 \pm 0.02$ & $21.3 \pm 0.9$ \\
${ }^{80} \mathrm{Se}$ & $0.02 \pm 0.03$ & $54.5 \pm 2.0$ \\
${ }^{82} \mathrm{Se}$ & $0.011 \pm 0.008$ & $9.3 \pm 0.4$ \\
Atomic weight, g mol ${ }^{-1}$ & $75.92 \pm 0.13$ & $78.96 \pm 0.03$ \\
${ }^{\text {a } 76}$ Se isotopic abundance of SeMet enriched with ${ }^{76} \mathrm{Se}$ (RM LGC 7330): $99.8(0.2) \%$ \\
\hline
\end{tabular}


Table 3. Selenium mass fractions ( $\mathrm{ng} \mathrm{g}^{-1} \mathrm{Se}$ ) determined in BCR-637 and SRM 1950 by postcolumn IDA-ICP-MS

\begin{tabular}{rccccc}
\hline \multirow{2}{*}{ Se-species } & \multicolumn{4}{c}{ BCR-637 } & SRM 1950 \\
\cline { 2 - 5 } & $\begin{array}{c}\text { This study } \\
(\mathrm{n}=7, k=2)\end{array}$ & $\begin{array}{c}{[15]^{\mathrm{a}}} \\
(\mathrm{n}=27)\end{array}$ & $\begin{array}{c}{[16]} \\
(\mathrm{n}=5)\end{array}$ & $\begin{array}{c}{\left[^{\mathrm{a}}\right]^{\mathrm{a}}} \\
(\mathrm{n}=3)\end{array}$ & $\begin{array}{c}\text { This study } \\
(\mathrm{n}=11, k=2)\end{array}$ \\
\hline GP×3 + non-retained species & $11.1 \pm 1.5$ & $16 \pm 3$ & $11 \pm 1$ & $13 \pm 1$ & $15.1 \pm 1.7$ \\
SEPP1 & $52.7 \pm 4.3$ & $51 \pm 5$ & $52 \pm 2$ & $64 \pm 5$ & $61.1 \pm 7.4$ \\
Se-Albumin & $15.8 \pm 2.5$ & $9 \pm 2$ & $17 \pm 2$ & $14 \pm 1$ & $23.1 \pm 3.7$ \\
Sum of species & $80 \pm 5$ & $75 \pm 9$ & $80 \pm 2$ & $90 \pm 5$ & $99 \pm 8$ \\
Total Se & & $79 \pm 7(k=2)^{\mathrm{a}}$ & & $105.5 \pm 3.8(k=2.2)$ \\
${ }^{\text {a }}$ Reported values have been converted into ng g \\
\hline
\end{tabular}




\section{Figures}

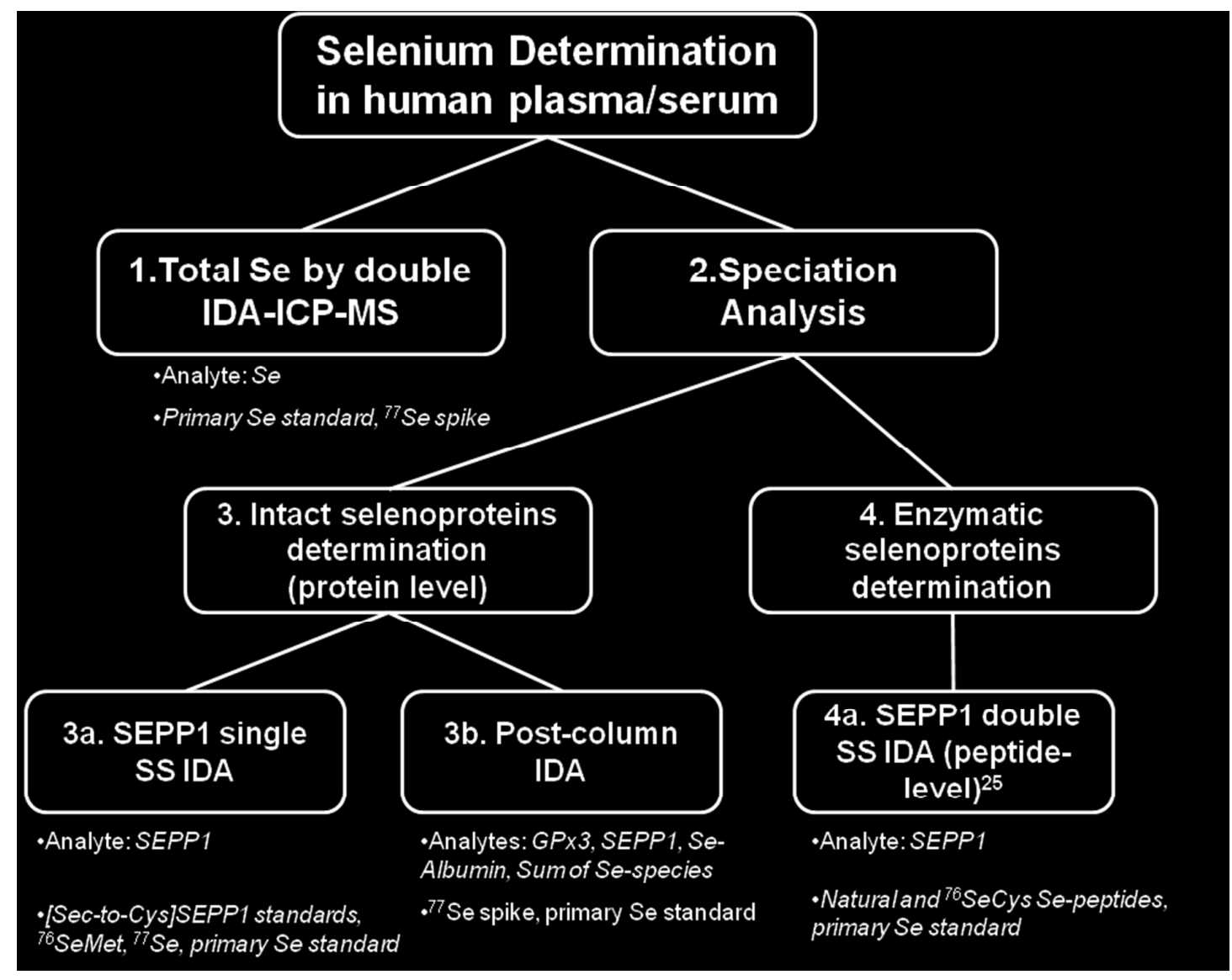

Figure 1. Workflow for the accurate assessment of selenoproteins in human serum 
A)
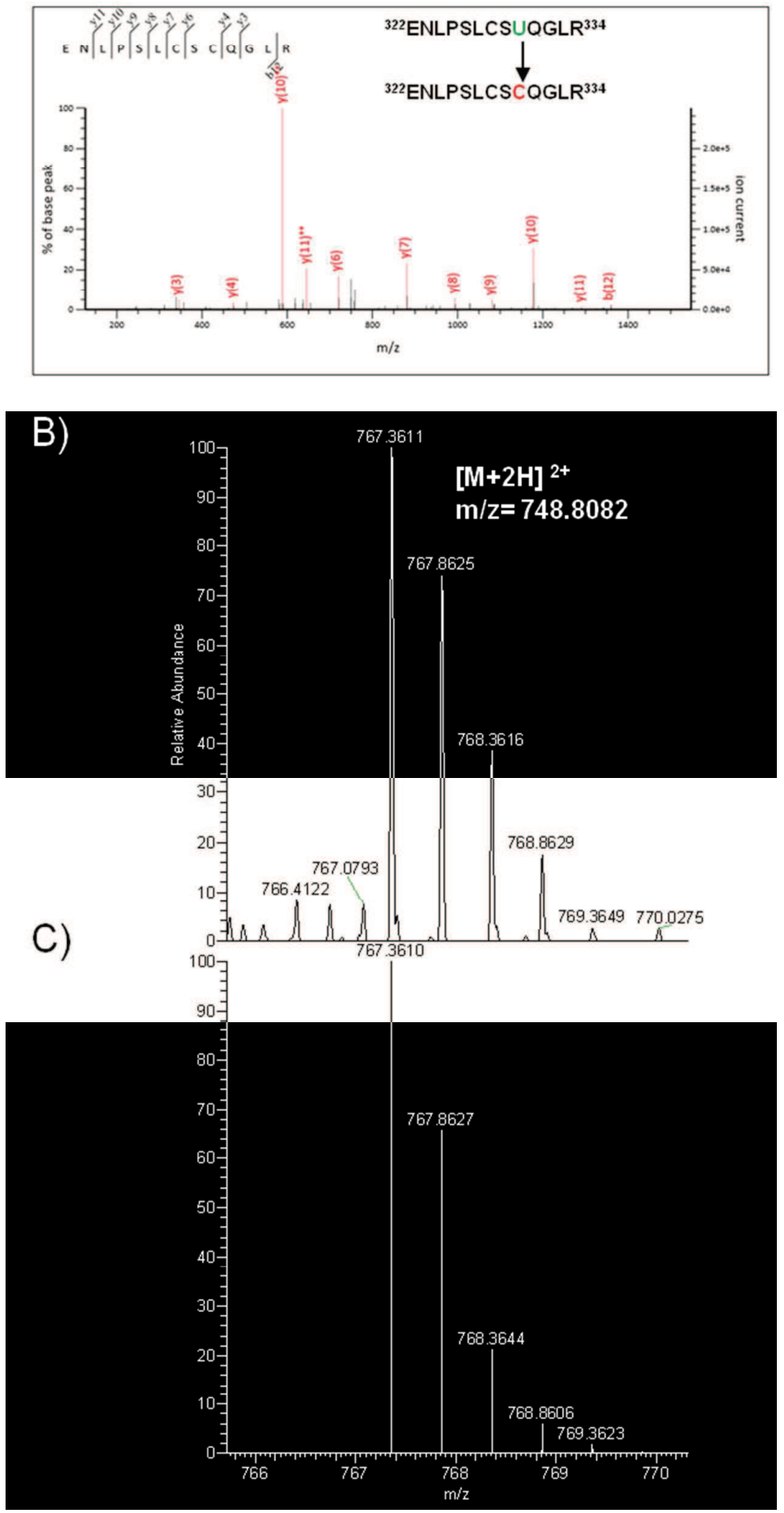

Figure 2. Molecular verification of the exchanged Cys residue $(C)$ for the [Sec-to-Cys]SEPP1 peptide ENLPSLCSCQGLR by ESI MS/MS. (a) MS/MS spectrum, (b) experimental and (c) theoretical isotope pattern. $\mathrm{U}=\mathrm{SeCys}$ 


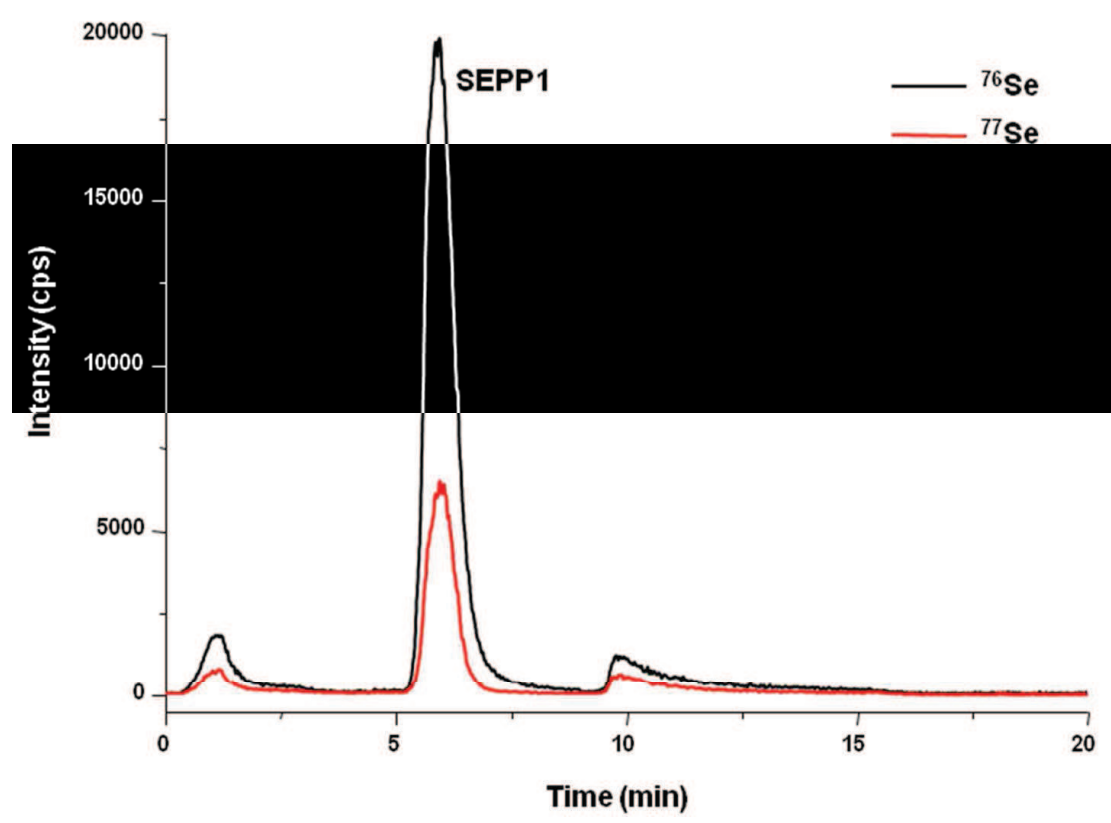

Figure 3. A sample blend (mixture of BCR-637 and the stable isotope enriched SEPP1 spike) chromatogram obtained by AF-HPLC-ICPMS 


\section{Supporting Information}

\section{Accurate quantification of selenoproteins in human plasma/serum by isotope dilution ICP-MS: Focus on Selenoprotein P}

M.Estela del Castillo Busto ${ }^{a}$, Caroline Oster ${ }^{a}$, Susana Cuello-Nuñez ${ }^{b}$, Christian L. Deitrich ${ }^{b}$, Andrea Raab ${ }^{c}$, Anna Konopka ${ }^{\mathrm{d}, \mathrm{e}}$, Wolf D. Lehmann ${ }^{\mathrm{d}}$, Heidi Goenaga-Infante ${ }^{\mathrm{b}}$ and Paola Fisicaro $^{*}$

\footnotetext{
a Laboratoire National de Métrologie et d'Essais (LNE), 1 rue Gaston Boissier, 75724 Paris Cedex 15, France. Email: paola.fisicaro@Ine.fr; Fax: +33140433737; Tel: +33140433759

${ }^{\mathrm{b}}$ LGC Limited, Queens Road, Teddington, TW11 OLY, Middlesex, United Kingdom.

${ }^{\mathrm{c}}$ TESLA University of Aberdeen, Department of Chemistry, Meston Walk, Aberdeen AB24 3UE, United Kingdom.

${ }^{\mathrm{d}}$ Molecular Structure Analysis, German Cancer Research Center (DKFZ), Im Neuenheimer Feld 280, 69120 Heidelberg, Germany.

${ }^{e}$ Faculty of Chemistry, Biological and Chemical Research Centre, University of Warsaw, Pasteura 1, 02-093 Warsaw, Poland.
}

Equation S-1. Model equation for species-specific IDA-ICP-MS for SEPP1

Equation S-2. Model equation for species-unspecific IDA-ICP-MS 
Equation S-1. Model equation for species-specific IDA-ICP-MS for SEPP1

The equation used for the determination of SEPP1 mass fraction (expressed as Se) in human plasma/serum by species-specific IDA-ICP-MS is shown below:

$w_{s}=w_{s p} \cdot \frac{m_{s p}}{m_{s}} \cdot \frac{W_{s}}{W_{s p}} \cdot \frac{\left(R_{A / B} \cdot k \cdot B_{s p}-A_{s p}\right)}{\left(A_{s}-R_{A / B} \cdot k \cdot B_{s}\right)}$

Equation 1

$w_{s}$, mass fraction of SEPP1 in the sample blend $\left(\mathrm{ng} \mathrm{g}^{-1} \mathrm{Se}\right)$

$w_{s p}$, mass fraction of SEPP1 in the spike ( $\left.\mathrm{ng} \mathrm{g}^{-1} \mathrm{Se}\right):(47.2 \pm 1.0) \mathrm{ng} \mathrm{g}^{-1} \mathrm{Se}, k=2$

$m_{s}$, mass of the sample in the sample blend $(\mathrm{g}):(0.10487 \pm 0.00051) \mathrm{g}, k=2$

$m_{s p}$, mass of the spike in the sample blend $(\mathrm{g}):(0.02567 \pm 0.00051) \mathrm{g}, k=2$

$W_{s}$, atomic weight of the sample $\left(\mathrm{g} \mathrm{mol}^{-1}\right):(78.96 \pm 0.03) \mathrm{g} \mathrm{mol}^{-1}$, IUPAC value, $k=2$

$W_{s p}$, atomic weight of the spike $\left(\mathrm{g} \mathrm{mol}^{-1}\right):(75.92 \pm 0.13) \mathrm{g} \mathrm{mol}^{-1}, k=2$

$R_{A / B}$, isotope ratio measured in the mixture: $R_{77 / 76}$

$k$, mass bias correction factor: $(0.8407 \pm 0.0088), k=2$

$A_{s}$, abundance of isotope $\mathrm{A}$ in the sample: $(7.635 \pm 0.010) \%$, IUPAC value

$B_{s}$, abundance of isotope $B$ in the sample: $(9.366 \pm 0.018) \%$, IUPAC value

$A_{s p}$, abundance of isotope $\mathrm{A}$ in the spike: $(0.03 \pm 0.02 \%, k=2)$

$B_{s p}$, abundance of isotope $B$ in the spike: $(99.85 \pm 0.05 \%, k=2)$ 
Equation S-2. Model equation for species-unspecific IDA-ICP-MS

The on-line equation used for the determination of the Se mass fraction bound to intact selenoproteins (GPx3, SEPP1 and Se-Albumin) in human serum by post-column IDA-AFHPLC-ICP-MS is shown below:

$M F_{\text {sample }}=w_{s p} \cdot f_{s p} \cdot \frac{W_{s}}{W_{s p}} \cdot \frac{\left(R_{A / B} \cdot k \cdot B_{s p}-A_{s p}\right)}{\left(A_{s}-R_{A / B} \cdot k \cdot B_{s}\right)}$

Equation 2

$M F_{\text {sample, }}$ mass flow of the Se (ng Se)

$w_{s p}$, mass fraction of the Se in the spike solution $\left(\mathrm{ng} \mathrm{g}^{-1} \mathrm{Se}\right):(0.504 \pm 0.017) \mathrm{ng} \mathrm{g}^{-1} \mathrm{Se}, k=2$

$f_{s p}$, flow of the spike solution $\left(\mathrm{g} \mathrm{min}^{-1}\right):(0.11005 \pm 0.00091) \mathrm{g} \mathrm{min}^{-1}, k=1$

$W_{s}$, atomic weight of the sample $\left(\mathrm{g} \mathrm{mol}^{-1}\right):(78.96 \pm 0.03) \mathrm{g} \mathrm{mol}^{-1}$, IUPAC value, $k=2$

$W_{s p}$, atomic weight of the spike $\left(\mathrm{g} \mathrm{mol}^{-1}\right):(76.92 \pm 0.03) \mathrm{g} \mathrm{mol}^{-1}, k=2$

$R_{A / B}$, isotope ratio measured in the mixture: $R_{76 / 77}$

$k$, mass bias correction factor: $(1.0431 \pm 0.0078), k=1$

$A_{s}$, abundance of isotope $\mathrm{A}$ in the sample: $(9.366 \pm 0.018) \%$, IUPAC value

$B_{s}$, abundance of isotope $B$ in the sample: $(7.635 \pm 0.010) \%$, IUPAC value

$A_{s p}$, abundance of isotope A in the spike: $(0.051 \pm 0.004) \%, k=2$

$B_{s p}$, abundance of isotope B in the spike: $(99.80 \pm 0.25) \%, k=2$

The mass fraction of $\mathrm{Se}\left(\mathrm{w}_{\text {sample }}, \mathrm{ng} \mathrm{g}^{-1} \mathrm{Se}\right)$ in the sample was obtained by the integration of the peak area divided by the mass injected $(\mathrm{g})$. The resulted mass fraction can be multiplied by the density of the serum $\left(\mathrm{g} \mathrm{mL}^{-1}\right)$ to get the Se concentration $\left(\mathrm{ng} \mathrm{mL} \mathrm{L}^{-1}\right)$ in each chromatographic peak.

$w_{\text {sample }}=\frac{\text { Area }}{m_{\text {injected }}} \quad$ or $\quad C_{\text {sample }}=\frac{\text { Area }}{m_{\text {injected }}} \cdot \rho_{\text {sample }}$

Equation 3

$C_{\text {sample, }}$ concentration of $\mathrm{Se}$ in the sample $\left(\mathrm{ng} \mathrm{mL}^{-1} \mathrm{Se}\right)$

$m_{\text {injected }}$, mass injected $(\mathrm{g}):(0.0495 \pm 0.0096) \mathrm{g}, k=2$

$\rho_{\text {sample }}$, density of BCR-637 RM $\left(\mathrm{g} \mathrm{mL}^{-1}\right):(1.0237 \pm 0.0044) \mathrm{g} \mathrm{mL}^{-1}, k=2$ 


\section{Accurate quantification of selenoproteins in human plasma/serum by isotope dilution ICP-MS: Focus on Selenoprotein P}

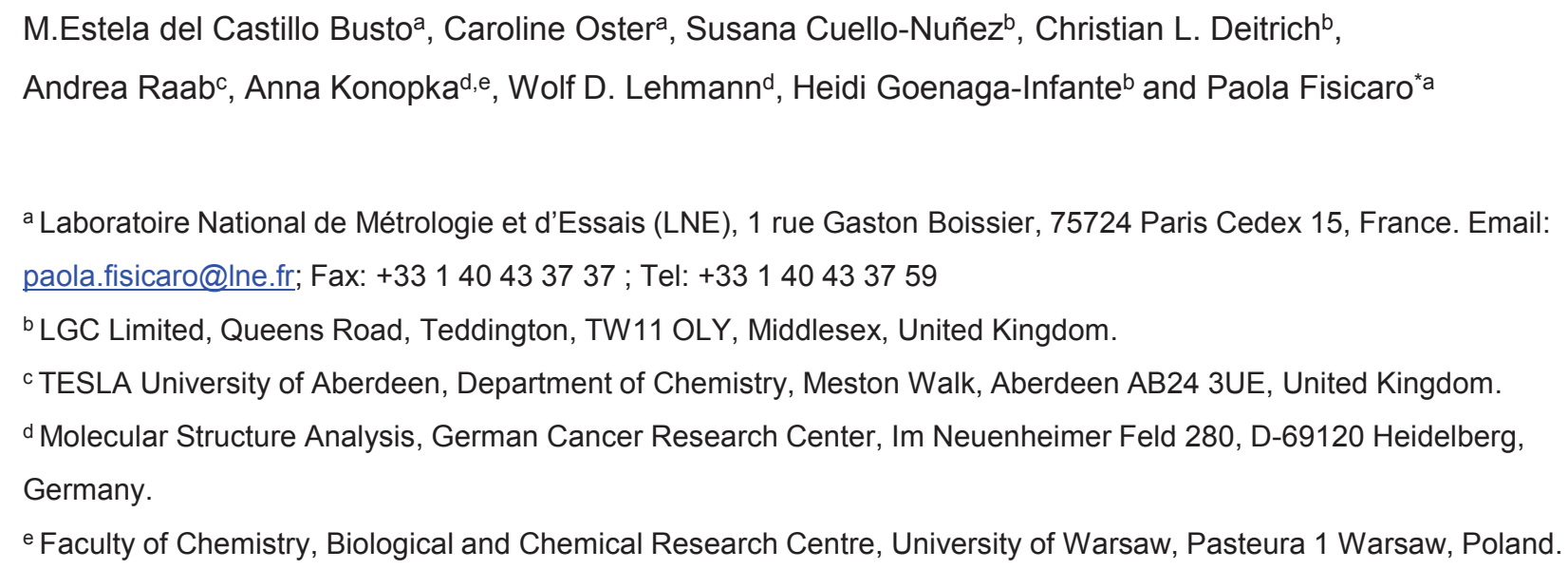

Figure 1. Workflow for the accurate assessment of selenoproteins in human serum

Figure 2. Molecular verification of the exchanged Cys residue (C) for the [Sec-toCys]SEPP1 peptide ENLPSLCSCQGLR by ESI MS/MS. (a) MS/MS spectrum, (b) experimental and (c) theoretical isotope pattern. $U=S e C y s$

Figure 3. A sample blend (mixture of BCR-637 and the stable isotope enriched SEPP1 spike) chromatogram obtained by AF-HPLC-ICPMS 


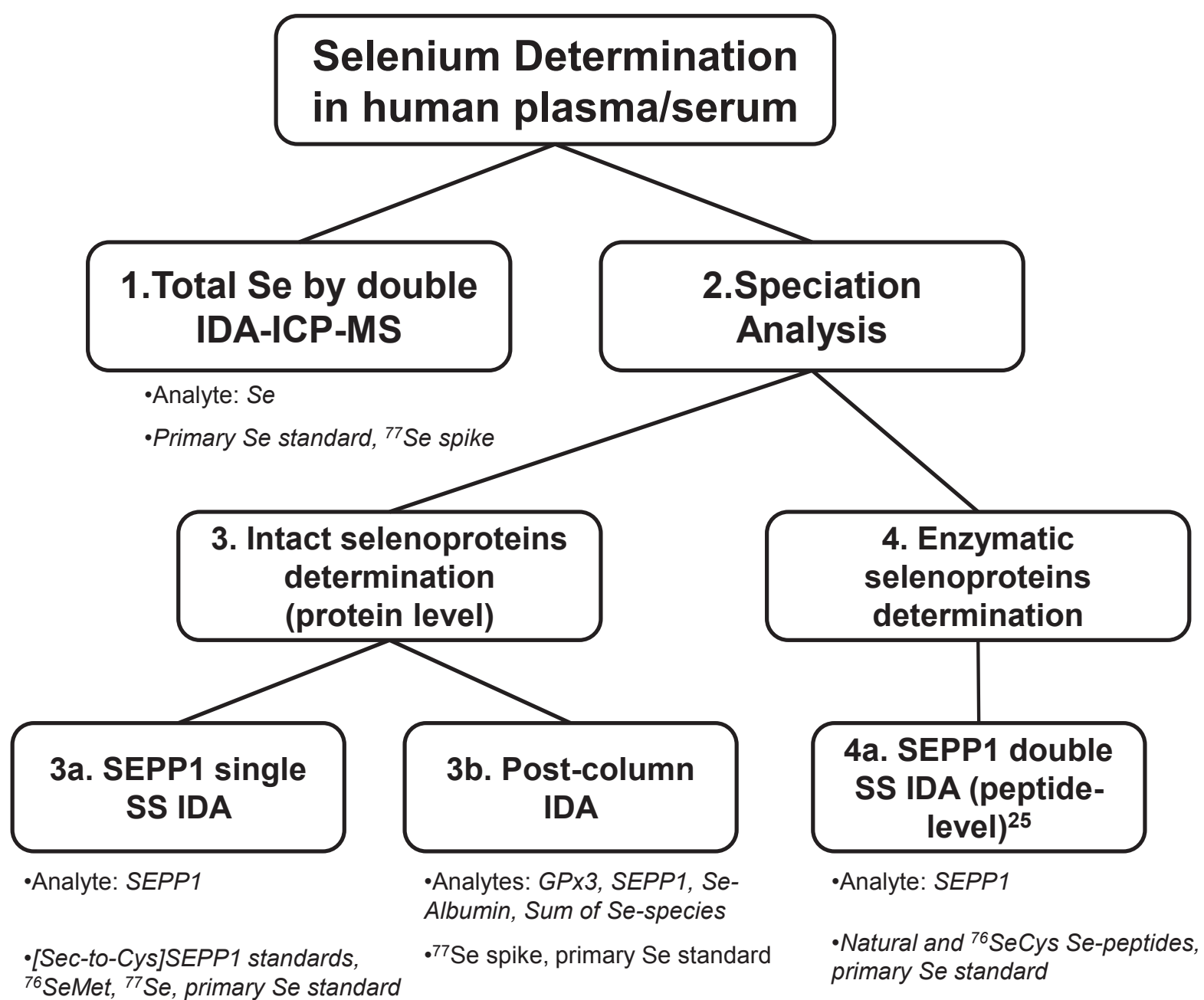

Figure 1. Workflow for the accurate assessment of selenoproteins in human serum. 


\section{Page 35 of 62}

A) Journal of Analytical Atomic Spectrometry

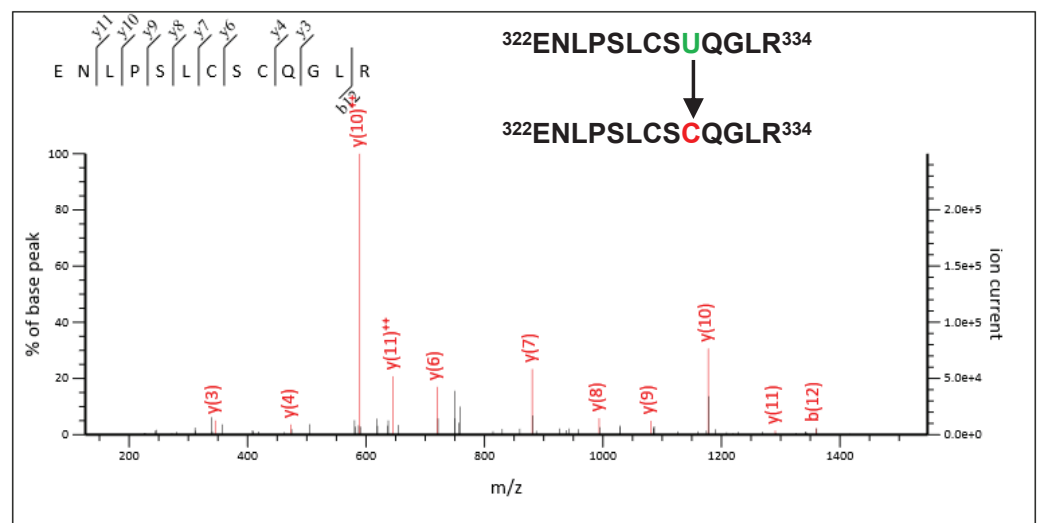

B)

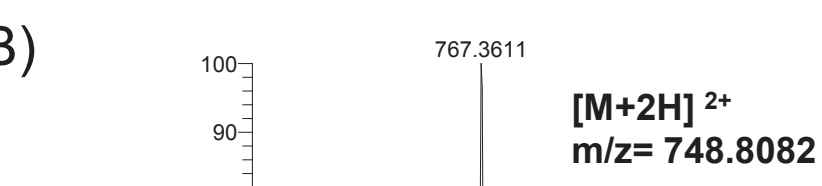

C)

768.3616

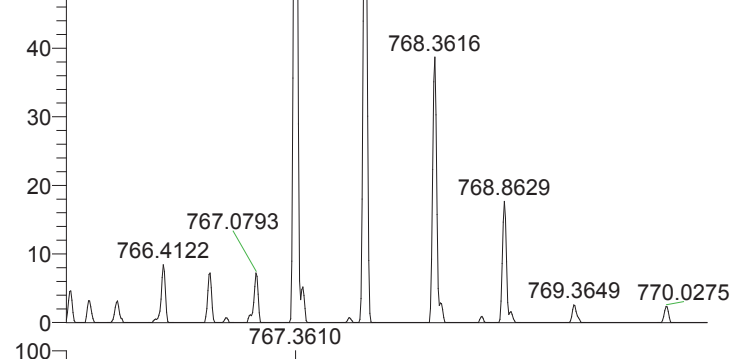

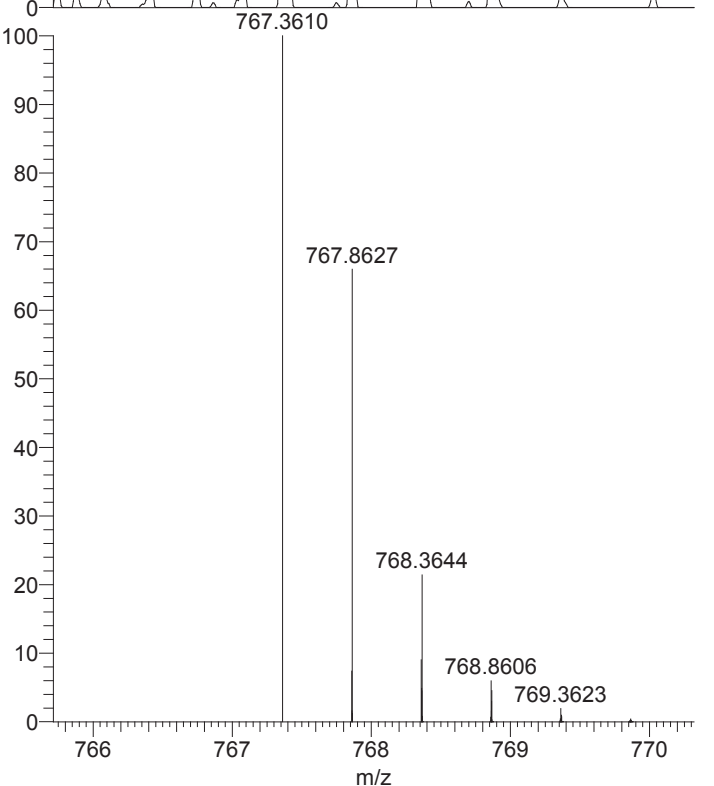

Figure 2. Molecular verification of the exchanged Cys residue $(C)$ for the [Sec-toCys]SEPP1 peptide ENLPSLCSCQGLR by ESI MS/MS. (a) MS/MS spectrum, (b) experimental and (c) theoretical isotope pattern. $\mathrm{U}=\mathrm{SeCys}$ 


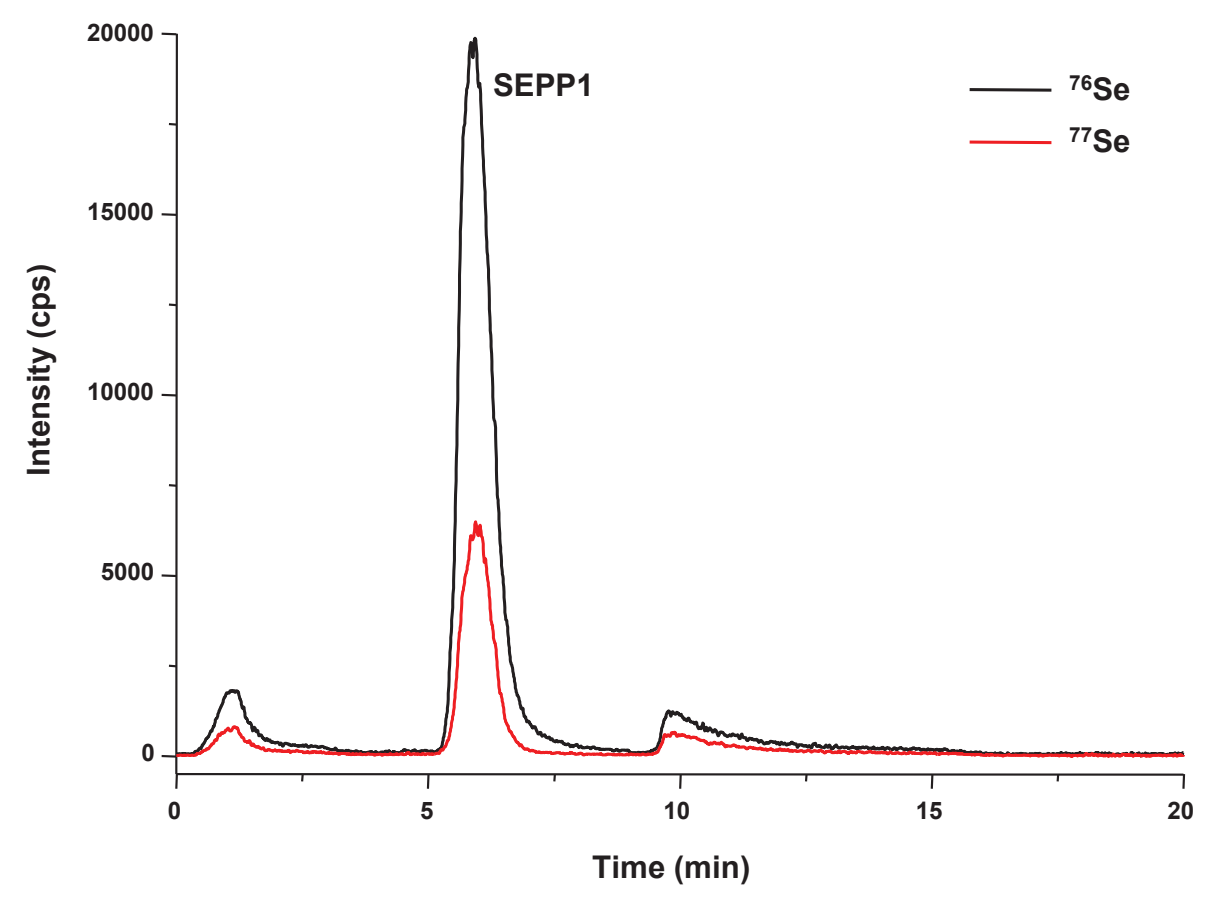

Figure 3. A sample blend (mixture of BCR-637 and the stable isotope enriched SEPP1 spike) chromatogram obtained by AF-HPLC-ICPMS 